\title{
ON THE PECULIARITIES OF X-RAY DETERMINATION OF CRYSTALLOGRAPHIC TEXTURE IN SAMPLES AFTER TESTING WEAR RESISTANCE
}

\author{
B.K. SOKOLOV ${ }^{\mathrm{a}, *}$, T.A. SOKOLOVA ${ }^{\mathrm{b}}$, L.P. VLADIMIROV ${ }^{\mathrm{a}}$, \\ L.I. ANISIMOVA ${ }^{\text {b }}$ and A.B. MAKAROV ${ }^{\mathrm{b}}$ \\ ${ }^{a}$ Institute of Metal Physics, Russian Academy of Sciences, 18 Kovalevskaya str., \\ 620219, Ekaterinburg, Russia; ' Institute of Machine Science, Russian Academy \\ of Sciences, 18 Kovalevskaya str., 620219, Ekaterinburg, Russia
}

(Received 4 February 2002; In final form 14 February 2002)

\begin{abstract}
A peculiar relief generally appears on the sample surface while testing wear resistance, which impairs the results of texture analysis by X-ray diffraction methods. The main reason for this is screening influence of the surface roughness on the X-ray passing through. From model representations, the influence on the screening of the relief parameters and the characteristic angle of reflection of X-rays is shown. Distribution of the parameters of a relief that actually forms is analyzed. A computer-assisted method of calculation of pole figures that excludes the screening effect is suggested. As an example of the method of application, the results of the analysis of textures that form upon testing wear resistance under condition of dry friction by slipping in pairs of samples of the $\beta$-titanium alloy VT35 doped with hydrogen are presented.
\end{abstract}

Keywords: Relief; Screening of X-rays; Pole figures

\section{INTRODUCTION}

Investigation of the texture changes upon different external actions helps in understanding the physical nature of phenomena and acting mechanisms of deformation and fracture. Depending on a majority

\footnotetext{
*Corresponding author.
} 
of different factors, the friction surface fracture in the course of testing wear resistance takes place while involving several main mechanisms (Bakly 1986; Veksler and Korshunov 1991). Available information on the texture changes in a substance is scarce, which is related, to a great extent, to complexity of studying texture in the samples under testing. The matter is that while testing wear resistance, on the sample surface, there arises a specific relief that consists of many grooves elongated in the direction of the sample or indentor movement (ED) (Fig. 1).

The central part of a direct pole figure of the sample with a relief has an unusual appearance (Fig. 2). Along ED, the pole density lowers rapidly from the center toward periphery, whereas along the transverse direction (TD) the pole density acquires constant values. Such a texture is similar to the axial one whose axis goes through ED.

To understand the origin of such a texture, it is necessary to analyze in more detail the geometrical conditions of X-ray examinations. Focusing upon examination is performed in accordance with the Bregg-Bretano's; experimental geometry was after Shults (reflection geometry) with a sample rotating around the normal to its surface (to $\beta$ angles) and turning around the stationary horizontal axis (to $\alpha$ angles) which lies in the plane of incident and reflected rays (honiometer plane). The intensity of reflection is fixed with a motionless counter. The direction of elongation of grooves ED corresponds to the value $\beta=0$ on the pole figure. In this sample position, the effect of screenings by the relief grooves is maximum at all $\alpha$ values, which

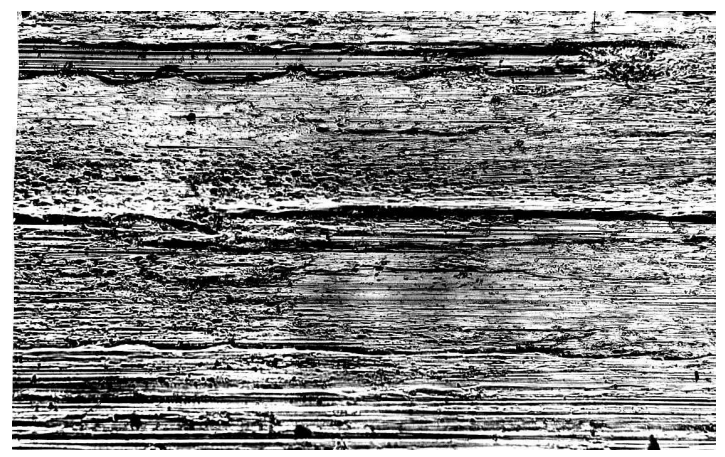

FIGURE 1 Relief on the surface of the VT35-alloy sample tested to wear resistance. Testing conditions are given in the text. Magnification -200 . 


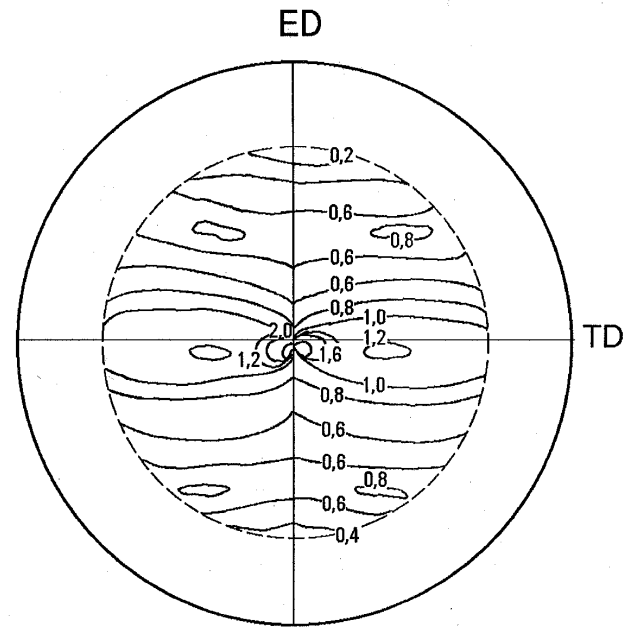

FIGURE 2 Central part of the pole figure $\{110\}$ taken from the relief sample. (For details see text).

manifests itself in lowering the reflected intensity. Upon turning the sample around the normal to its surface, the screening effect decreases, reaching a minimum value in the TD direction at $\beta=90^{\circ}$.

Besides the screening effect, the relief gives rise to a total lowering of the reflected intensity because of focusing. Another disadvantage is impossibility of experimenting in the transmission geometry and taking complete pole figures caused by the practical shortcomings when preparing foils of equal thickness from the surface layers of the relief sample.

\section{MODELING OF THE SCREENING EFFECT}

To ascertain the regularities of manifestation of the screening effect, the sample relief was simulated by a row of repeating grooves in the form of triangular prisms with equal parameters.

Of great significance for the determination of screening conditions are the parameters of equilateral triangles in the prism cross-section: length of the triangle base $\left(a_{0}\right)$ and its height $\left(h_{0}\right)$. Subscript 0 means that the values refer to the sample cross-section. To characterize the relief, it is more convenient to use the ratio $a_{0} / 2 h_{0}$ which is equal to 
$\operatorname{arctg} \delta_{0}$, where $\delta_{0}$ is half angle at the apex of the prism cross-section. Figure 3 schematically shows the relief contours in the groove crosssection and the direction of X-rays incident at a characteristic angle $\theta$. In case (a), the relief parameters are such that $\delta_{0}$ is larger than the angle $(90-\theta)$. In this case, all the incident rays are reflected. However, if $\delta_{0}$ is smaller that $(90-\theta)$, as in case (b), the majority of $\mathrm{X}$-rays does not come into the counter. Expressions:

$$
\delta>\left(90^{\circ}-\theta_{0}\right), \operatorname{arctg}\left(a_{0} / 2 h_{0}\right)>\left(90^{\circ}-\theta\right)
$$

are the conditions for the absence of the screening effect.

With changes in the direction of elongation of the relief grooves relative to the goniometer plane (the sample turning to the angles $\alpha$ and $\beta$ ), the shape of the prism cross-section modifies, as well as the length of bases and heights. Calculation allowed us to derive a general expression of the dependence of $\delta$ on polar coordinates of the groove elongation direction on the stereographic projection.

$$
\delta=\operatorname{arctg} \frac{a_{0} \cdot \cos \alpha}{2 h_{0} \sin \left(90^{\circ}-\beta\right)}
$$

Expressions (1) and (2) make it possible to calculate on the polar stereographic projection positions of the regions in which the screening effect does not manifest itself.

Figure 4 represents the polar stereographic projection with drawn boundaries of the regions in which the screening effect is absent for the relief parameters $\delta_{0}=65^{\circ}$ or $a_{0} / 2 h_{0}=2.14$ and a number of characteristic angles of reflection $(\theta)$ equal to 23,33 , and $42^{\circ}$. Regions from
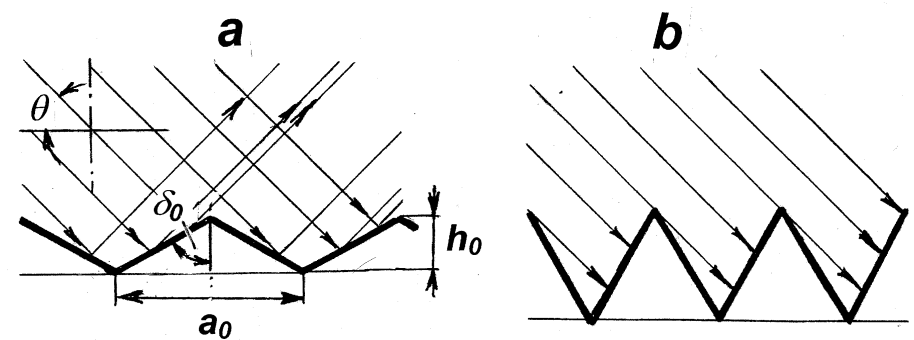

FIGURE 3 Contours of cross-sections of a model relief and directions of the incident and reflected X-rays. 
TD to curves are free of screening. One can see that with increasing $\theta$ angle the size of regions where the screening effect is observed decreases. Consequently, measurements at large angles $\theta$ reduce the screening effect of relief on the form of pole figure.

The influence of the relief parameters in the cross-section on the size of regions free of screening at one and the same angle $\theta$ is shown in Fig. 5.

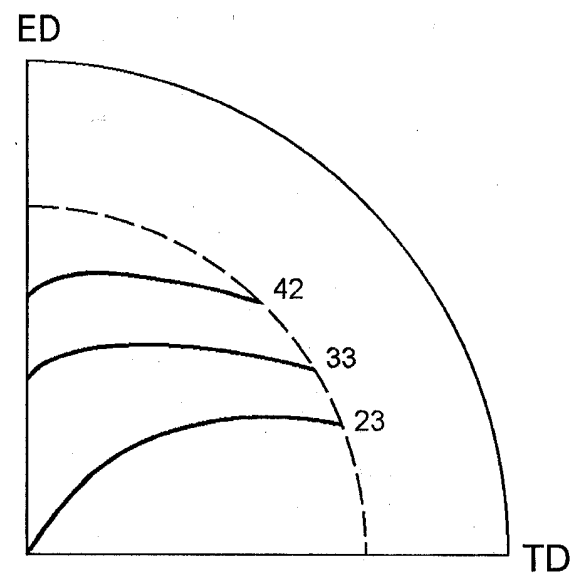

FIGURE 4 Stereographic projection with the drawn regions in which the screening effect is absent. Digits show characteristic angles $(\theta)$ of the X-ray reflection; $\delta_{0}=65^{\circ}$.

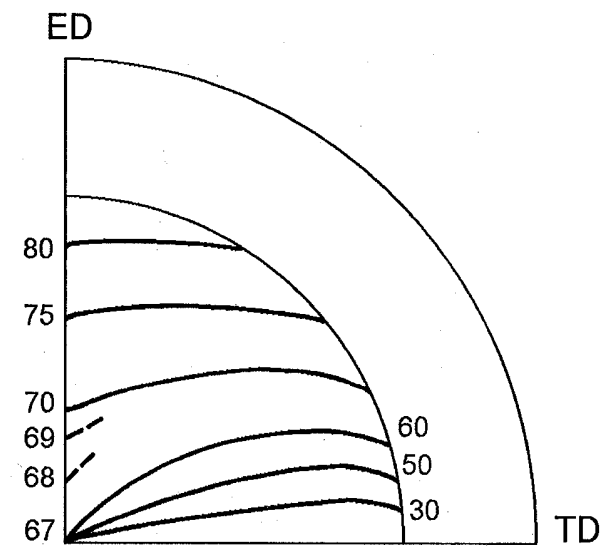

FIGURE 5 Stereographic projection with the drawn regions in which the screening effect is absent. Digits show the values of the angle $\theta$ which characterize the relief parameters; $\theta=23^{\circ}$. 
Digits give the angle value $\delta_{0}$. The more the angle $\delta_{0}$, the less the screening effect influence the form of a pole figure. It should be noted that if $\delta_{0}<(90-\theta)$, the loss of the pole density occurs in the center of the pole figure.

\section{DISTRIBUTION OF THE RELIEF PARAMETERS IN REAL SAMPLES}

The real relief on the sample surface when testing wear resistance is a statistic distribution of elongated grooves of different shapes and parameters. Figure 6(a) shows a profilegram of the relief for the sample tested for wear resistance upon dry friction in a like pair. One can see that along with a coarse corrugation (the height of coarse grooves reached $140 \mu \mathrm{m}$ ) there takes place fine ripples (on the order of $5-10 \mu \mathrm{m})$. The width of grooves changes in broad limits as well.

Quantitative description of the real relief is given by the histogram of distribution of the grooves parameters over $\operatorname{arctg} a_{0}^{\prime} / h_{0}^{\prime}$ (Fig. 6b) where $a_{0}^{\prime}$ is length of the horizontal projection of a portion tilted at an angle $\delta_{0}^{\prime} ; h_{0}^{\prime}$ is the roughness height. The superscript (') distinguishes the real values from the model ones.

On the ordinate axis, the quantity $n=\sum a_{\delta^{\prime}} / N$, where $\sum a_{\delta^{\prime}}$ is the sum of lengths of horizontal projections of portions with a certain angle $\delta_{0}^{\prime}$, and $N$ is the total length of all the portions of the relief cross-section under measurement.

In the samples tested to wear resistance upon dry friction regions having $\delta_{0}^{\prime}=88-90^{\circ}$ take up $20-30 \%$, and $\delta_{0}^{\prime}<60^{\circ}, 10-15 \%$.

\section{ALLOWANCE FOR THE SCREENING EFFECT IN CONSTRUCTING POLE FIGURES}

The density of the reflected intensity for every point on the pole figure is controlled first by crystallographic conditions of the textured state and, second, by the above-considered effect of screenings. It is necessary to correct the pole figure so that to exclude the screening effect.

The histogram shown in Fig. 6(b) allows determination of the relative part of X-ray radiation that is received by each group of the relief grooves with definite angles $\delta_{0}^{\prime}$. It is proportional to the value $n$. 
(a) $\mathbf{h}_{0, \mu \mathrm{m}}^{\prime}$

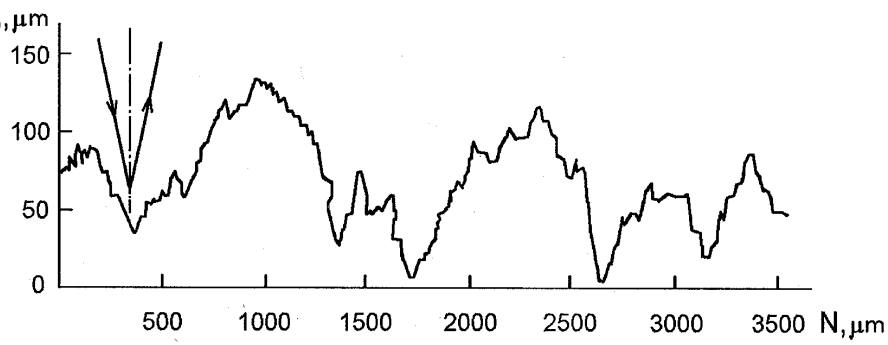

(b)

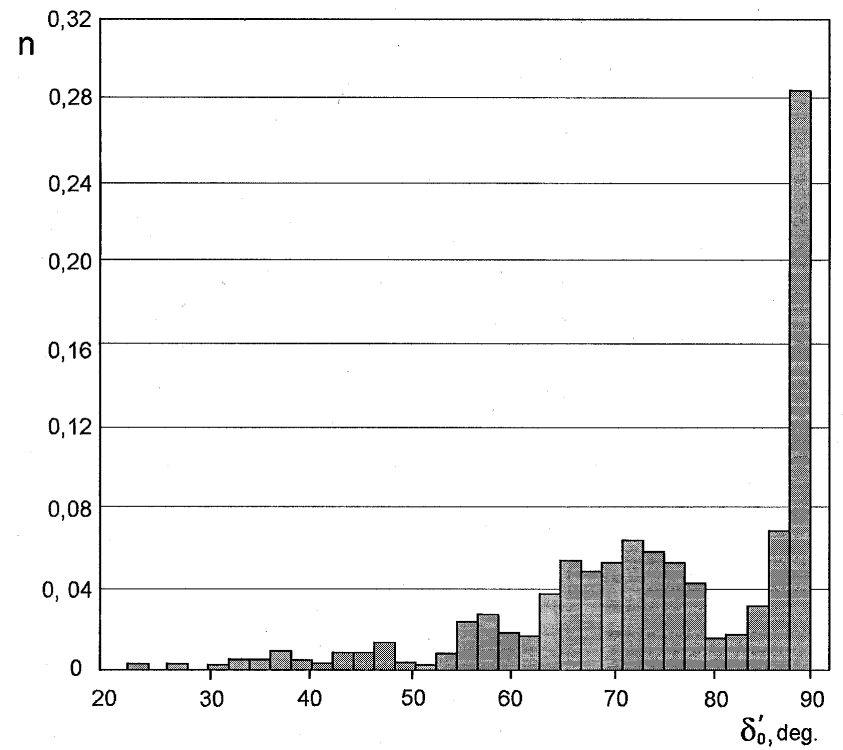

FIGURE 6 (a) Profilegram of cross-section of the sample relief after testing to wear resistance; (b) histogram of distribution of the relief parameters over the angles $\delta_{0}^{\prime}$.

For a chosen radiation (angle $\theta$ ) and the values $\delta_{0}^{\prime}$ obtained from histograms, one can construct the stereographic projection with marked contours of regions in which the screening effect is absent. In accordance with the histogram, each region has its own value $n$. If a point on the stereographic projection belongs at the same time to several regions in which the screening effect is absent, the values $n$ are summed up for them.

To normalize the pole density in each region of the pole figure, experimental data are to be divided by the corresponding value $n$. 
In our experiment, X-ray measurements were performed on a diffractometer DRON-2 with a goniometer GYR-8 and an automatic attachment. Samples of $\beta$-titanium alloy VT35 were studied in Co $\mathrm{K}_{\alpha}$ radiation with integrated back-and-forth motion of a sample.

The automatic attachment sequentially rotates the sample around the normal to its plane (angle $\beta$ ) by given angular steps. At each turn, the reflected X-ray intensity was collected and fixed during a definite exposure time. After complete turning by $\beta=360^{\circ}$ the sample was tilted with respect to the horizontal axis by a given angle $\alpha$ and the sample again was successively rotated over the whole interval of $\beta$ angles and so forth up to $\alpha=70^{\circ}$. A computer program provided the working regimes and intensity recording for each angular $\alpha$ and $\beta$ positions. $\beta$-angle steps were set $5^{\circ}$ and the time of the intensity collecting was $6 \mathrm{~s}$. The computer program allowed taking into account the reflection background and calculation of the relative intensity density in comparison with the textureless state.

Correction of the pole figure was carried out as described above. The relief profile was determined in the points on the sample surface that were distant from each other by $10 \mu \mathrm{m}$ along transverse directions by the light-focusing method on a setup PMT-3 with a magnification of 600 . The accuracy of measurements of the relief height was $2 \mu \mathrm{m}$.

The data resulted from the relief measurements were inputed in the computer program that allows construction of the histogram of the relief roughness distribution and calculation of the angles $\alpha$ and $\beta$ that characterize the contours of regions, in which the screening effect is absent, together with the corresponding values $n$. The latter operation of the computer program is the data correction that serves to exclude the screening effect and construct the corrected pole figure.

\section{APPLICATION OF THE METHOD DEVELOPED TO STUDY THE INFLUENCE OF HYDROGEN CONTENT IN $\beta$-TITANIUM ALLOY ON THE TEXTURE FORMED UPON TESTING WEAR RESISTANCE}

This investigation is a part of work on the influence of hydrogen on the level of mechanical and tribological properties of $\beta$-titanium alloy VT35 with bec lattice (Makarov et al., 2002). The alloy had 
the following composition: $\mathrm{Al}-2.7, \mathrm{Mo}-0.55, \mathrm{~V}-15.2, \mathrm{Cr}-3$, $\mathrm{Zr}-0.4, \mathrm{Sn}-2.8$. He is mechanically stable; the $\beta-\alpha$ transformation proceeds in it solely after rather long holdings at temperatures 300-500 ${ }^{\circ} \mathrm{C}$ (Moder et al., 1992).

Sheets of industrial alloy $1.2 \mathrm{~mm}$ thick were annealed in vacuum at $800^{\circ} \mathrm{C}$ and then hydrogenated from the gaseous phase at $900^{\circ} \mathrm{C}$ and different exposures, which resulted in different hydrogen concentrations in the samples from 0.005 to $0.37 \mathrm{wt} . \%$. Average grain size in the samples made up $450 \mu \mathrm{m}$. Texture in the samples was absent. The method of measuring the integral X-ray intensity allowed one to reveal an insignificant enhancement of the $\{110\}\langle\mathrm{uvw}\rangle$-type component (level 1.4 with respect to the textureless sample) and of the $\{222\}\langle\mathrm{uvw}\rangle$ one (level 1.2).

Testing was performed under conditions of dry friction by slipping in a like pair sample-plate. The average slipping rate upon back-and-forth motion of the sample was $0.021 \mathrm{~m} / \mathrm{s}$; the length of working stroke was $12 \mathrm{~mm}$, the number of double runs, 1000, the normal load, $196 \mathrm{~N}$. Testing atmosphere was argon.

The pole figure $\{110\}$ shown in Fig. 2 was obtained from the sample containing $0.37 \mathrm{wt} \%$ hydrogen after testing wear resistance. The relief section and histogram in Fig. 6 also refer to the same sample. Qualitatively, the same appearance was represented by the pole figures, reliefs and their histograms for the samples differing in the hydrogen content as well. However, there occurred some quantitative differences.

The corrected pole figure of the sample with $0.37 \mathrm{wt} \%$ hydrogen is shown in Fig. 7. It is seen that upon testing wear resistance there forms very weak texture. Of the most noticeable texture components, the scattered $\{110\}\langle 001\rangle$ ones can be picked out (the maximum pole density 1.5 times exceeds the level of the initial sample) as well as the $\{112\}\langle 111\rangle$ (maximum pole density 1.3 times as high as in the initial sample). The pole density of the $\{111\}\langle\mathrm{uvw}\rangle$ component decreased remarkably. Similar regularities manifest themselves also in the pole figure taken from the sample with a relief when using the reflections $\{220\}$ (the angle $\theta$ is equal to $44^{\circ}$, which significantly lowers the screening effect manifestation).

These pole figures represent an asymmetry relative to the transverse direction. From the doublet of orientations of the type $\{112\}\langle 111\rangle$ only one is pronounced. 


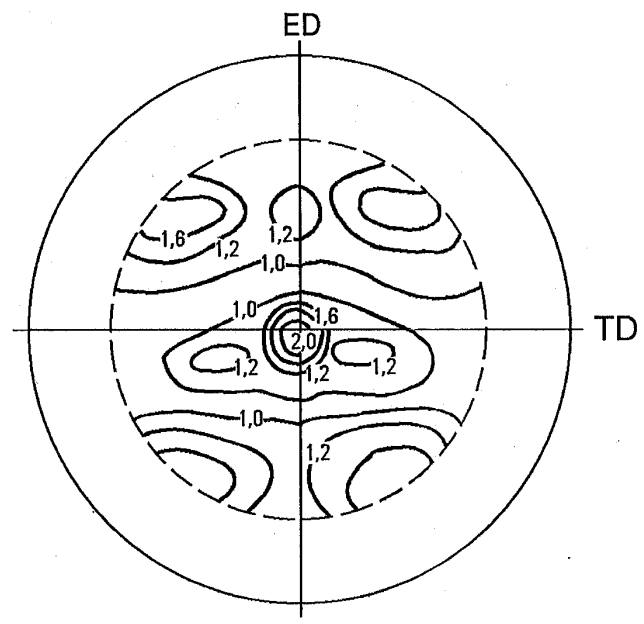

FIGURE 7 Central part of the pole figure $\{110\}$ of the sample with the relief shown in Fig. 2 after its correction.

The asymmetry of texture as well as the enhancement of the component noted is typical of the friction textures. Thus, in the work (Sidorov et al., 1983), a substantial development of the above component in the texture of the BCC-Fe-3wt.\% Si alloy after cold rolling to $53 \%$ is shown (the friction coefficient is $0.2-0.3$ ). In many works on the rolling with large friction coefficients, the asymmetry of the pole figures obtained was noted as well (for example, Lee and Duggan, 1991).

Changes in the hydrogen concentration in the alloy insignificantly and quantitatively affect the texture character. With increasing the hydrogen concentration, the pole density of the $\{110\}\langle 001\rangle$ component rises from the intensity level of 1.5 to 2.0 . The pole density of $\{112\}\langle 111\rangle$ is retained constant.

Maximum azimuthal width of the region with the pole density 1.4 around the component of the type $\{110\}\langle 001\rangle$ makes up about $50^{\circ}$ for the alloys with $0.1-0.2$ hydrogen, which is significantly higher than in alloys without hydrogen or with $0.37 \mathrm{wt} . \%$ hydrogen (about $\left.25-35^{\circ}\right)$.

In a number of works it was noted that in bec metals and alloys, detection of the texture in the course of testing of wear resistance is hindered, which was related to a large number of systems of slipping 
(for example, Babareko, 1969). In our experimental conditions, the above factors did not suppress the texture formation but it was discovered only while taking into account the effect of screening of X-rays.

\section{CONCLUSIONS}

The results of the study presented allow one to make the following conclusions.

1. The screening influence of the relief arising upon testing wear resistance on the friction texture that is determined in registration of the reflected X-rays is detected.

2. Based on model representations, the dependence of the screening degree on the relief parameters such as depth and width of grooves as well as the value of angle $\theta$ of incident radiation is determined. The regions of stereographic projection in which the screening effect is absent are calculated.

3. The corrected pole figures were obtained from the sample surface after testing under conditions of friction by slipping $\beta$ titanium alloys doped with hydrogen. It is established that the friction textures formed are asymmetrical relative to the transverse direction of elongation of the relief grooves and are represented by the components of the type $\{110\}\langle 001\rangle$ or $\{112\}\langle 111\rangle$ of weak intensity. With increasing the hydrogen concentration in the alloy, the component $\{110\}\langle 001\rangle$ is somewhat enhanced.

4. The results of work can be used to analyze texture in the samples with reliefs obtained by other different methods.

\section{References}

Babareko, L.A. (1969). Development of texture in metals and alloys upon deformation and recrystallization. Itogi nauki i tekhniki. In: Metallovedenie $i$ termoobrabotka, pp. 5-83. Viniti, Moscow.

Bakli, D. (1986). Surface phenomena upon adhesion and friction interaction, 359p. Mashinostroenie, Moscow.

Lee, C.S. and Duggan, D.J. (1991). A simple theory for the development of inhomogeneous rolling textures. Metall. Trans., 22A(11), 2637-2641.

Makarov, A.V., Anisimova, L.I., Korshunov, L.G., Sokolova, T.A. and Sokolov, B.K. (2002). The influence of large hydrogen concentrations $(0.1-0.65 \mathrm{wt} . \%)$ on 
tribological properties of titanium alloy VT35. Trudy Ural' skoi shkoly metallovedov, p. 218. Nauka-servis, Ufa.

Moder, N.I., Moiseev, V.N. and Antipov, A.I. et al. (1992). Transformations upon isothermal treatment of pseudo-binary titanium alloy VT35. Metallovedenie $i$ termoobrabotka metallov, 3, 22-25.

Sidorov, V.A., Sokolov, B.K. and Bukhvalov, O.B. (1983). Peculiarities of texture of silicon iron that is formed upon rolling with intense contact friction. Izv. AN USSR (ser. metally), 3, 122-124.

Vecsler, Yu. G. and Korshunov, B.G. (1991). Metal testing to wear resistance upon friction. In: under edition of Bernshtein M.L., Metallovedenie $i$ termicheskaya obrabotka stali, p. 462. Metallurgia, Moscow. 